PROCEEDINGS OF THE

AMERICAN MATHEMATICAL SOCIETY

Volume 136, Number 12, December 2008, Pages 4245-4255

S 0002-9939(08)09523-3

Article electronically published on July 29, 2008

\title{
EIGENVALUE ESTIMATES FOR MAGNETIC SCHRÖDINGER OPERATORS IN DOMAINS
}

\author{
RUPERT L. FRANK, ARI LAPTEV, AND STANISLAV MOLCHANOV \\ (Communicated by Mikhail Shubin)
}

\begin{abstract}
Inequalities are derived for sums and quotients of eigenvalues of magnetic Schrödinger operators with non-negative electric potentials in domains. The bounds reflect the correct order of growth in the semi-classical limit.
\end{abstract}

\section{Introduction AND MAIN RESUlts}

In this paper we derive inequalities for the eigenvalues of magnetic Schrödinger operators in a domain. Such inequalities have received a lot of attention in the case where both the electric and the magnetic potential are absent. If $0<\lambda_{1}^{(0)}<\lambda_{2}^{(0)} \leq$ ... denote the eigenvalues of the Dirichlet Laplacian in a domain $\Omega \subset \mathbb{R}^{d}$ of finite measure, then the Berezin-Li-Yau inequality [B, LY] states that

$$
\sum_{j}\left(\lambda-\lambda_{j}^{(0)}\right)_{+} \leq \frac{2}{d+2} v_{d}|\Omega| \lambda^{1+d / 2}
$$

for any $\lambda>0$. Here

$$
v_{d}:=\left|\mathbb{S}^{d-1}\right| / d=\pi^{d / 2} / \Gamma(1+d / 2)
$$

denotes the volume of the $d$-dimensional unit ball. Performing a Legendre transform in (1.1) one obtains (see [LW])

$$
\sum_{j=1}^{k} \lambda_{j}^{(0)} \geq \frac{4 \pi^{2} d}{d+2} v_{d}^{-2 / d}|\Omega|^{-2 / d} k^{1+2 / d} .
$$

An important feature of inequalities (1.1) and (1.3) is that they are sharp in the semi-classical limit. Indeed, according to Weyl's theorem (see, e.g., [LL, RS])

$$
\lambda_{k}^{(0)} \sim 4 \pi^{2} v_{d}^{-2 / d}|\Omega|^{-2 / d} k^{2 / d}, \quad k \rightarrow \infty,
$$

which implies sharpness of (1.1) as $\lambda \rightarrow \infty$ and of (1.3) as $k \rightarrow \infty$.

Given the lower bounds (1.1) and (1.3) on eigenvalue sums it is natural to ask for upper bounds which have the correct asymptotic behavior. In $\mathrm{L}$ it is shown

Received by the editors May 29, 2007.

2000 Mathematics Subject Classification. Primary 35P15; Secondary 35J10.

Key words and phrases. Eigenvalue bounds, semi-classical estimates, Laplace operator, magnetic Schrödinger operator.

(c) 2008 by the authors. This paper may be reproduced, in its entirety, for non-commercial purposes. 
that

$$
\sum_{j}\left(\lambda-\lambda_{j}^{(0)}\right)_{+} \geq \frac{2}{(d+2)(2 \pi)^{d}} v_{d}\|\omega\|_{\infty}^{-2}\left(\lambda-\lambda_{1}^{(0)}\right)_{+}^{1+d / 2}
$$

for all $\lambda>0$, where $\omega \geq 0$ denotes the normalized eigenfunction corresponding to the eigenvalue $\lambda_{1}^{(0)}$. Later it was observed in $[\mathrm{H}]$ that by an isoperimetric inequality of Chiti [C], $\|\omega\|_{\infty}$ can be bounded from above in terms of the eigenvalue $\lambda_{1}^{(0)}$. This yields the estimate

$$
\sum_{j}\left(\lambda-\lambda_{j}^{(0)}\right)_{+} \geq \frac{2}{d+2} H_{d}^{-1}\left(\lambda_{1}^{(0)}\right)^{-d / 2}\left(\lambda-\lambda_{1}^{(0)}\right)_{+}^{1+d / 2}
$$

with a non-sharp but explicit constant

$$
H_{d}:=\frac{2 d}{j_{(d-2) / 2}^{2} J_{d / 2}^{2}\left(j_{(d-2) / 2}\right)} .
$$

Here $J_{\nu}$ denotes the Bessel function of order $\nu$ and $j_{\nu}$ its first positive zero. Passing to the Legendre transform one finds

$$
\sum_{j=1}^{k}\left(\lambda_{j}^{(0)}-\lambda_{1}^{(0)}\right) \leq \frac{d}{d+2} H_{d}^{2 / d} \lambda_{1}^{(0)} k^{1+2 / d} .
$$

Both inequalities (1.6) and (1.8) reflect the correct order of growth with respect to $k$ as given by (1.4). Note, however, that $\lambda_{1}^{(0)}$ in these inequalities replaces $|\Omega|^{-2 / d}$ in (1.1) and (1.3). Bounds (1.6) and (1.8) can be used to derive upper bounds on the eigenvalue ratios $\lambda_{k} / \lambda_{1}$ from (1.6) and (1.8). The sharp constant in the case $k=2$ was found in $\mathrm{AB} 3$.

The purpose of the present paper is to show that inequality (1.6) and its corollaries extend, with the same constants, to the case of a non-trivial magnetic field and a non-negative electric potential. More precisely, let $\Omega \subset \mathbb{R}^{d}, d \geq 2$, be a domain of finite measure, let $V \in L_{1, \text { loc }}(\Omega)$ be non-negative and let $A \in L_{2, \text { loc }}\left(\Omega, \mathbb{R}^{d}\right)$ be arbitrary. We define the Schrödinger operator

$$
H:=(D-A)^{2}+V \quad \text { in } L_{2}(\Omega)
$$

by closing its quadratic form on $C_{0}^{\infty}(\Omega)$. We assume that this operator has compact resolvent and denote its eigenvalues by $0<\lambda_{1} \leq \lambda_{2} \leq \ldots$, where each eigenvalue is repeated according to multiplicities.

Our main result is

Theorem 1.1. For any $\lambda \geq 0$ one has

$$
\sum_{j}\left(\lambda-\lambda_{j}\right)_{+} \geq \frac{2}{d+2} H_{d}^{-1} \lambda_{1}^{-d / 2}\left(\lambda-\lambda_{1}\right)_{+}^{1+d / 2}
$$

with the constant $H_{d}$ from (1.7). Moreover, for any $k \in \mathbb{N}$ one has

$$
\sum_{j=1}^{k}\left(\lambda_{j}-\lambda_{1}\right) \leq \frac{d}{d+2} H_{d}^{2 / d} \lambda_{1} k^{1+2 / d} .
$$

From these estimates on sums of eigenvalues one easily obtains estimates on the ratio $\lambda_{k+1} / \lambda_{1}$. 
Corollary 1.2. For any $k \in \mathbb{N}$ one has

$$
\begin{gathered}
\lambda_{k+1} \leq\left(1+\left(1+\frac{d}{2}\right)^{2 / d} H_{d}^{2 / d} k^{2 / d}\right) \lambda_{1}, \\
\lambda_{k+1} \leq\left(1+\frac{4}{d}\right)\left(1+\frac{d}{d+2} H_{d}^{2 / d} k^{2 / d}\right) \lambda_{1} .
\end{gathered}
$$

We emphasize that both estimates in Corollary 1.2 have the correct order of growth as $k \rightarrow \infty$. Indeed, asymptotics (1.4) are also valid for the eigenvalues $\lambda_{k}$, provided $V, A$ and $\Omega$ are sufficiently regular.

For large values of $k$, the bound (1.12) is better than (1.11). For small values of $k$, (1.11) is better than (1.12), but an even better bound for small values of $k$ is given by

$$
\lambda_{k+1} \leq\left(1+\frac{4}{d}\right)^{k} \lambda_{1}, \quad k \in \mathbb{N} .
$$

The latter is a Payne-Pólya-Weinberger-type bound which is also derived below. For a further discussion of the different constants in bounds (1.11), (1.12) and (1.13), we refer to $\mathrm{H}$.

We emphasize that all our bounds are known in the case $V \equiv A \equiv 0$; see $[\mathrm{H}]$. Our point here is that they extend, with the same constants, to the case of a non-trivial magnetic field and a non-negative electric potential. This is non-trivial since the diamagnetic inequality (see, e.g., [LL, Thm. 7.21]) gives only information about the lowest eigenvalue or about exponential sums of eigenvalues. In particular, it seems unclear to us whether the PPW-conjecture (see AB3]), namely that the quotient $\lambda_{2} / \lambda_{1}$ is maximized for a circle and $V \equiv 0$, extends to the magnetic case.

We remark that our inequalities are also true if $d=1$, but in this case much stronger results are known. (Recall that any magnetic field in one dimension can be gauged away.) In particular, if $V \geq 0$, then one has the sharp inequality $\lambda_{k} / \lambda_{1} \leq k^{2}$; see $\mathrm{AB} 2$. A beautiful proof of this inequality for $k=2$ is given in [AB1].

\section{Proof of Theorem 1.1}

2.1. Main ingredients. Our proof of Theorem 1.1 is based on three propositions which might be of independent interest. We defer their proofs to the following sections. First, we derive a lower bound on the eigenvalue sum $\sum_{j}\left(\lambda-\lambda_{j}\right)_{+}$in terms of $\lambda$ and the maximum of the lowest eigenfunction.

Proposition 2.1. Let $\omega$ be any eigenfunction corresponding to the lowest eigenvalue $\lambda_{1}$ of $H$ with $\|\omega\|=1$. Then for any $\lambda \geq 0$ one has

$$
\sum_{j}\left(\lambda-\lambda_{j}\right)_{+} \geq \frac{2 v_{d}}{(d+2)(2 \pi)^{d}}\|\omega\|_{\infty}^{-2}\left(\lambda-\lambda_{1}\right)_{+}^{1+d / 2}
$$

with $v_{d}$ given in (1.2).

In the case $V \equiv A \equiv 0$, this is Theorem 4.1 in $\mathrm{L}$. It turns out that almost the same proof holds even for arbitrary $A$ and $V \geq 0$. We emphasize that in the magnetic case the lowest eigenvalue $\lambda_{1}$ may be degenerate and $\|\omega\|_{\infty}$ may depend on the choice of the eigenfunction. Some inequalities in the same spirit have been obtained in $[\mathrm{S}]$ for elliptic differential operators with variable coefficients. 
In order to use Proposition 2.1 for the proof of Theorem 1.1 we need to estimate the maximum of the lowest eigenfunction in terms of the corresponding eigenvalue.

Proposition 2.2. Let $\lambda$ be an eigenvalue of $H$ and $\omega$ a corresponding eigenfunction. Then for any $p>0$

$$
\|\omega\|_{\infty} \leq C_{d}(p) \lambda^{d / 2 p}\|\omega\|_{p}
$$

with the universal constant

$$
C_{d}(p)=\left(2^{p(d-2) / 2} \Gamma(d / 2)^{p}\left|\mathbb{S}^{d-1}\right| \int_{0}^{j_{(d-2) / 2}} J_{(d-2) / 2}^{p}(r) r^{-p(d-2) / 2+d-1} d r\right)^{-1 / p} .
$$

In particular,

$$
C_{d}(2)=\left(\pi^{d / 2} 2^{d-2} \Gamma(d / 2) j_{(d-2) / 2}^{2} J_{d / 2}^{2}\left(j_{(d-2) / 2}\right)\right)^{-1 / 2} .
$$

In the case $V \equiv A \equiv 0$, this is a result of Chiti C]. Non-negative electric potentials can be included without major changes, as was done in AB3. Using Kato's inequality we shall show that the same proof even works for a non-trivial magnetic field.

We emphasize that (2.2) is an isoperimetric result: If $\Omega$ is a ball, $V \equiv \operatorname{curl} A \equiv 0$ and $\lambda$ is the lowest eigenvalue of $H$, then one has equality in (2.2). This explains the value of the constant $C_{d}(p)$. Discussing the cases of equality would be beyond the scope of this paper.

The third ingredient employed in the proof of our main results is the following Yang-type inequality.

Proposition 2.3. For any $k \in \mathbb{N}$ one has

$$
\sum_{j=1}^{k}\left(\lambda_{k+1}-\lambda_{j}\right)\left(\lambda_{k+1}-\left(1+\frac{4}{d}\right) \lambda_{j}\right) \leq 0 .
$$

This result follows from Theorem 5 in $[\mathrm{HS}$ (see also $\mathrm{LP}$ for results in the same spirit). For the sake of completeness we include an independent variational proof which is closer to Yang's original argument $[\mathrm{Y}]$ in the case $V \equiv A \equiv 0$ (see also [A, CY].).

From Proposition 2.3 one can deduce several weaker but more explicit eigenvalue estimates.

Corollary 2.4. For any $k \in \mathbb{N}$ one has

(Y) $\lambda_{k+1} \leq\left(1+\frac{4}{d}\right) \frac{1}{k} \sum_{j=1}^{k} \lambda_{j}$

(HP) $\frac{1}{k} \sum_{j=1}^{k} \frac{\lambda_{j}}{\lambda_{k+1}-\lambda_{j}} \geq \frac{d}{4}$

(PPW) $\lambda_{k+1}-\lambda_{k} \leq \frac{4}{d} \frac{1}{k} \sum_{j=1}^{k} \lambda_{j}$.

In the case $V \equiv A \equiv 0$ these estimates are known as second Yang inequality, Hile-Protter inequality and Payne-Pólya-Weinberger inequality, respectively. For any fixed $k$ one has the implications

$$
(2.4) \Rightarrow(\mathrm{Y}) \Rightarrow(\mathrm{HP}) \Rightarrow(\mathrm{PPW}) \text {. }
$$

The proof of (2.5) is based on general arguments (see [A]) and will not be reproduced here. 
Remark 2.5. Note that (PPW) implies (1.13). Indeed, estimating $\frac{1}{k} \sum_{j=1}^{k} \lambda_{j} \leq \lambda_{k}$ we obtain $\lambda_{k+1} / \lambda_{k} \leq 1+4 / d$. Multiplying these estimates for consecutive values of $k$ we obtain (1.13).

2.2. Proof of the main results. We now show how Theorem 1.1 and Corollary 1.2 follow from Propositions 2.1, 2.2 and 2.3.

Proof of Theorem 1.1. To get (1.9) we simply combine estimates (2.1) and (2.2) and note that $H_{d}=(2 \pi)^{d} v_{d}^{-1} C_{d}(2)^{2}$.

To deduce (1.10) from (1.9) we use the Legendre transform argument from [LW]. Indeed, for a function $h$ on $[0, \infty)$ put $L h(p):=\sup _{\lambda>0}(p \lambda-h(\lambda))$. So if $f(\lambda):=$ $\sum_{j}\left(\lambda-\lambda_{j}\right)_{+}$and $g(\lambda):=\frac{2}{d+2} H_{d}^{-1} \lambda_{1}^{-d / 2}\left(\lambda-\lambda_{1}\right)_{+}^{1+d / 2}$, then

$$
L f(p)=\{p\} \lambda_{[p]+1}+\sum_{j=1}^{[p]} \lambda_{j}, \quad L g(p)=p \lambda_{1}+\frac{d}{d+2} H_{d}^{2 / d} \lambda_{1} p^{1+2 / d}
$$

(with $\{p\},[p]$ the fractional and integer parts of $p$ ). Now $f \geq g$ implies $L f \leq L g$, and evaluating the latter at an integer $p=k \in \mathbb{N}$ we obtain (1.10).

Proof of Corollary 1.2, For the proof of (1.11) we put $\lambda=\lambda_{k+1}$ in (1.9) and estimate $\sum_{j=1}^{k}\left(\lambda_{k+1}-\lambda_{j}\right) \leq k\left(\lambda_{k+1}-\lambda_{1}\right)$. For the proof of (1.11) we combine (Y) with (1.10).

\section{UPPER BOUND ON EIGENVALUE SUMS}

In this section we extend the argument of $[\mathrm{L}$ to prove Proposition 2.1. We let $u_{1}:=\omega$ and denote by $u_{j}, j \in \mathbb{N}$, orthonormal eigenfunctions corresponding to the eigenvalues $\lambda_{j}$. We put $\theta_{\xi}(x):=e^{i \xi \cdot x} \omega(x), \tilde{\omega}:=\|\omega\|_{\infty}$ and calculate using Plancherel's equality

$$
\begin{aligned}
\sum_{j}\left(\lambda-\lambda_{j}\right)_{+} & \geq \tilde{\omega}^{-2} \sum_{j}\left(\lambda-\lambda_{j}\right)_{+} \int_{\Omega}\left|\bar{\omega} u_{j}\right|^{2} d x \\
& =(2 \pi)^{-d} \tilde{\omega}^{-2} \sum_{j}\left(\lambda-\lambda_{j}\right)_{+} \int_{\mathbb{R}^{d}}\left|\int_{\Omega} \bar{\omega}(x) u_{j}(x) e^{-i \xi \cdot x} d x\right|^{2} d \xi \\
& =(2 \pi)^{-d} \tilde{\omega}^{-2} \int_{\mathbb{R}^{d}} \sum_{j}\left(\lambda-\lambda_{j}\right)_{+}\left|\left(\theta_{\xi}, u_{j}\right)\right|^{2} d \xi \\
& =(2 \pi)^{-d} \tilde{\omega}^{-2} \int_{\mathbb{R}^{d}}\left(\int_{0}^{\infty}(\lambda-\nu)_{+} d\left(E(\nu) \theta_{\xi}, \theta_{\xi}\right)\right) d \xi
\end{aligned}
$$

Here $d E(\nu)$ denotes the spectral measure of $H$. We note that

$$
\int_{0}^{\infty} d\left(E(\nu) \theta_{\xi}, \theta_{\xi}\right)=\left\|\theta_{\xi}\right\|^{2}=\|\omega\|^{2}=1
$$

Since the function $\nu \mapsto(\lambda-\nu)_{+}$is convex, we can apply Jensen's inequality to get

$$
\begin{aligned}
\int_{0}^{\infty}(\lambda-\nu)_{+} d\left(E(\nu) \theta_{\xi}, \theta_{\xi}\right) & \geq\left(\lambda-\int_{0}^{\infty} \nu d\left(E(\nu) \theta_{\xi}, \theta_{\xi}\right)\right)_{+} \\
& =\left(\lambda-\int_{\Omega}\left(\left|(D-A) \theta_{\xi}\right|^{2}+V\left|\theta_{\xi}\right|^{2}\right) d x\right)_{+} .
\end{aligned}
$$

We shall need the following 
Lemma 3.1. For any $\xi \in \mathbb{R}^{d}$ one has

$$
\int_{\Omega}\left(\left|(D-A) \theta_{\xi}\right|^{2}+V\left|\theta_{\xi}\right|^{2}\right) d x=\lambda_{1}+|\xi|^{2} .
$$

Proof. Denoting $h[u]=\int_{\Omega}\left(|(D-A) u|^{2}+V|u|^{2}\right) d x$, an easy calculation shows that

$$
h\left[\theta_{\xi}\right]=\lambda_{1}+|\xi|^{2}+2 j \cdot \xi, \quad j:=\operatorname{Im} \int_{\Omega} \bar{\omega}(\nabla-i A) \omega d x
$$

and we have to show that $j=0$. Assume to the contrary that $j \neq 0$ and choose $\xi_{0}$ such that $j \cdot \xi_{0}<0$. Then in view of (3.4), $h\left[\theta_{\varepsilon \xi_{0}}\right]<\lambda_{1}$ for all sufficiently small $\varepsilon>0$. But this contradicts the variational characterization of the lowest eigenvalue.

Combining (3.1), (3.2) and (3.3) we arrive at

$$
\begin{aligned}
\sum_{j}\left(\lambda-\lambda_{j}\right)_{+} & \geq(2 \pi)^{-d} \tilde{\omega}^{-2} \int_{\mathbb{R}^{d}}\left(\lambda-\lambda_{1}-|\xi|^{2}\right)_{+} d \xi \\
& =(2 \pi)^{-d} \tilde{\omega}^{-2} v_{d} \frac{2}{d+2}\left(\lambda-\lambda_{1}\right)_{+}^{1+d / 2}
\end{aligned}
$$

which proves the assertion.

\section{Eigenfunction ESTIMATES FOR MAGNetic SchröDinger OPERATORS}

4.1. A non-sharp bound. As we pointed out after Proposition 2.2, the constants in (2.2) are sharp. Before giving its rather involved proof we would like to derive a (non-sharp) estimate which still has the correct form needed in the proof of Theorem 1.1. More precisely, we shall prove that if $\omega$ is an eigenfunction corresponding to an eigenvalue $\lambda$ of $H$, then

$$
\|\omega\|_{\infty} \leq \tilde{C}_{d} \lambda^{d / 4}\|\omega\|_{2}, \quad \tilde{C}_{d}=(e / d \pi)^{d / 4} .
$$

To see this, we note that by the diamagnetic inequality and the Feynman-Kac formula [D] one has for all $t>0$ and all $x \in \Omega$ the pointwise estimate

$$
\left|e^{-t \lambda} \omega(x)\right|=\left|\left(e^{-t H} \omega\right)(x)\right| \leq\left(e^{t \Delta}|\omega|\right)(x) .
$$

Here $|\omega|$ is extended by 0 to $\mathbb{R}^{d}$ and $e^{t \Delta}$ denotes the heat semi-group on $\mathbb{R}^{d}$. Using the explicit expression of its kernel we can estimate

$$
\left(e^{t \Delta}|\omega|\right)(x) \leq(4 \pi t)^{-d / 2}\left(\int_{\mathbb{R}^{d}} e^{-|x-y|^{2} / 2 t} d y\right)^{1 / 2}\|\omega\|_{2}=(4 \pi t)^{-d / 4}\|\omega\|_{2} .
$$

Combining the previous two relations and optimizing with respect to $t$ we arrive at

$$
|\omega(x)| \leq \inf _{t>0} e^{t \lambda}(4 \pi t)^{-d / 4}\|\omega\|_{2}=(e / d \pi)^{d / 4} \lambda^{d / 4}\|\omega\|_{2} .
$$


4.2. Proof of Proposition 2.2. Now we turn to the sharp bound (2.2). We introduce

$$
S:=\left\{x \in \mathbb{R}^{d}:|x|<j_{(d-2) / 2} \lambda^{-1 / 2}\right\}, \quad z(x):=|x|^{-(d-2) / 2} J_{(d-2) / 2}(\sqrt{\lambda}|x|) .
$$

Note that $S$ is the ball centered at the origin for which the Dirichlet problem (with $V \equiv A \equiv 0$ ) has lowest eigenvalue $\lambda$. The function $z$ is the corresponding eigenfunction. Next, let

$$
\Omega^{*}:=\left\{x \in \mathbb{R}^{d}:|x|<v_{d}^{-1 / d}|\Omega|^{1 / d}\right\}
$$

be the ball centered at the origin with the same volume as $\Omega$ (with $v_{d}$ from (1.2)).

Lemma 4.1. One has $S \subset \Omega^{*}$.

Proof. We denote by $\lambda_{1}(-\Delta, \mathcal{O})$ the lowest eigenvalue of the Dirichlet Laplacian on a domain $\mathcal{O}$. Note that by definition of $S, \lambda_{1}(-\Delta, S)=\lambda$. The variational principle, together with the diamagnetic inequality and the non-negativity of $V$, implies $\lambda \geq \lambda_{1}(-\Delta, \Omega)$. Finally, the Faber-Krahn inequality states that $\lambda_{1}(-\Delta, \Omega) \geq$ $\lambda_{1}\left(-\Delta, \Omega^{*}\right)$. Hence we have $\lambda_{1}(-\Delta, S) \geq \lambda_{1}\left(-\Delta, \Omega^{*}\right)$, which by the variational principle implies $S \subset \Omega^{*}$.

We denote by $\omega^{*}: \Omega^{*} \rightarrow[0, \infty)$ the spherically decreasing rearrangement of $\omega$ (see [LL]) and by $u$ the function on $[0,|\Omega|]$ such that

$$
u\left(v_{d}|x|^{d}\right)=\omega^{*}(x), \quad x \in \Omega^{*} .
$$

Note that $u$ is decreasing with $u(0)=\|\omega\|_{\infty}$ and $u(|\Omega|)=0$. It satisfies the following integro-differential inequality.

Lemma 4.2. For u defined in (4.2) one has

$$
-u^{\prime}(s) \leq d^{-2} v_{d}^{-2 / d} \lambda s^{-2+2 / d} \int_{0}^{s} u(t) d t, \quad 0<s<|\Omega| .
$$

Proof. Kato's inequality [S1, [S2] states that $\operatorname{Re}\left((\operatorname{sgn} w)(D-A)^{2} w\right) \geq-\Delta|w|$. Here $\operatorname{sgn} w(x)=\overline{w(x)} /|w(x)|$ if $w(x) \neq 0$ and $\operatorname{sgn} w(x)=0$ otherwise. Since $V \geq 0$ we have

$$
\lambda|\omega|=\operatorname{Re}\left((\operatorname{sgn} \omega)\left((D-A)^{2}+V\right) \omega\right) \geq-\Delta|\omega| .
$$

We integrate this inequality over the set $\{|\omega|>t\}$ and use Gauss's theorem in the form

$$
-\int_{\{|w|>t\}} \Delta|w| d x=\int_{\{|w|=t\}}|\nabla| w|| d \sigma .
$$

Here $d \sigma$ denotes $(d-1)$-dimensional surface measure. We obtain

$$
\lambda \int_{\{|\omega|>t\}}|\omega| d x \geq \int_{\{|\omega|=t\}}|\nabla| \omega|| d \sigma .
$$

To estimate the integral on the RHS from below, we introduce the distribution function

$$
\mu(t):=|\{x \in \Omega:|\omega|>t\}|
$$

and recall the co-area formula

$$
-\mu^{\prime}(t)=\int_{\{|\omega|=t\}}|\nabla| \omega||^{-1} d \sigma
$$


Hence by Cauchy-Schwarz

$$
\begin{aligned}
\sigma(\{|\omega|=t\})^{2} & \leq\left(\int_{\{|\omega|=t\}}|\nabla| \omega||^{-1} d \sigma\right)\left(\int_{\{|\omega|=t\}}|\nabla| \omega|| d \sigma\right) \\
& =-\mu^{\prime}(t) \int_{\{|\omega|=t\}}|\nabla| \omega|| d \sigma .
\end{aligned}
$$

Because of the isoperimetric inequality

$$
\sigma(\{|\omega|=t\}) \geq d v_{d}^{1 / d} \mu(t)^{1-1 / d}
$$

we arrive at

$$
\int_{\{|\omega|=t\}}|\nabla| \omega|| d \sigma \geq d^{2} v_{d}^{2 / d} \mu(t)^{2-2 / d}\left(-\mu^{\prime}(t)\right)^{-1} .
$$

Combining this with (4.4) we have shown that

$$
\mu(t)^{2-2 / d}\left(-\mu^{\prime}(t)\right)^{-1} \leq d^{-2} v_{d}^{-2 / d} \lambda \int_{\{|\omega|>t\}}|\omega| d x .
$$

It remains to substitute $t=u(s)$ and to note that when $u(s) \neq 0$,

$$
\mu(t)^{2-2 / d}\left(-\mu^{\prime}(t)\right)^{-1}=s^{2-2 / d}\left(-u^{\prime}(s)\right), \quad \int_{\{|\omega|>t\}}|\omega| d x=\int_{0}^{s} u(r) d r .
$$

This proves the assertion.

This allows us to sketch the

Proof of Proposition 2.2. Following the proof of [C] Thm. 1] word by word, with his Lemmas 1 and 2 replaced by our Lemmas 4.1 and 4.2. we obtain the pointwise inequality $\omega^{*} \geq z$ in $S$ under the normalization $\|\omega\|_{L_{\infty}(\Omega)}=\|z\|_{L_{\infty}(S)}$. Then we can follow the proof of [C, Thm. 2] to obtain our Proposition 2.2.

\section{Yang inequalities for magnetic Schrödinger operators}

We turn now to the proof of Proposition 2.3. As before we denote by $u_{i}, i \in \mathbb{N}$, orthonormal eigenfunctions corresponding to the eigenvalues $\lambda_{i}$. Throughout the proof we will fix $k \in \mathbb{N}$. Moreover, for any $1 \leq i \leq k$ and any $1 \leq l \leq d$ we define the function

$$
\varphi_{i}:=x_{l} u_{i}-\sum_{j=1}^{k} a_{i j} u_{j}, \quad a_{i j}:=\int x_{l} u_{i} \overline{u_{j}} d x
$$

(suppressing the dependence on $l$ in the notation for the moment) and note that

$$
\left(\varphi_{i}, u_{j}\right)=0, \quad 1 \leq j \leq k .
$$

Hence the variational principle implies

$$
\lambda_{k+1} \leq \frac{\int\left(\left|(D-A) \varphi_{i}\right|^{2}+V\left|\varphi_{i}\right|^{2}\right) d x}{\int\left|\varphi_{i}\right|^{2} d x}
$$

(with the convention that the RHS is infinite if $\varphi_{i} \equiv 0$ ). Using the commutator identity

$$
\left[(D-A)^{2}, x_{l}\right]=-2 \mathrm{i}\left(D_{l}-A_{l}\right)
$$


and the orthogonality (5.1) one easily finds

$$
\int\left(\left|(D-A) \varphi_{i}\right|^{2}+V\left|\varphi_{i}\right|^{2}\right) d x=\lambda_{i} \int\left|\varphi_{i}\right|^{2} d x+2 \operatorname{Im} \int\left(D_{l}-A_{l}\right) u_{i} \overline{\varphi_{i}} d x
$$

which, together with (5.2), leads to

$$
\lambda_{k+1}-\lambda_{i} \leq \frac{2 \operatorname{Im} \int\left(D_{l}-A_{l}\right) u_{i} \overline{\varphi_{i}} d x}{\int\left|\varphi_{i}\right|^{2} d x} .
$$

Next, we manipulate the numerator. We note that

$$
\begin{aligned}
2 \operatorname{Im} \int\left(D_{l}-A_{l}\right) u_{i} \overline{x_{l} u_{i}} d x & =-2 \operatorname{Re} \int \frac{\partial u_{i}}{\partial x_{l}} \overline{x_{l} u_{i}} d x=-\int x_{l} \frac{\partial\left|u_{i}\right|^{2}}{\partial x_{l}} d x \\
& =\int\left|u_{i}\right|^{2} d x=1,
\end{aligned}
$$

and hence

$$
2 \operatorname{Im} \int\left(D_{l}-A_{l}\right) u_{i} \overline{\varphi_{i}} d x=1+2 \operatorname{Re} \sum_{j=1}^{k} \overline{a_{i j}} b_{i j},
$$

where we have set

$$
b_{i j}:=\mathrm{i} \int\left(D_{l}-A_{l}\right) u_{i} \overline{u_{j}} d x
$$

With the help of (5.3) we calculate

$$
2 b_{i j}=-\int\left(x_{l} u_{i} \overline{(D-A)^{2} u_{j}}-(D-A)^{2} u_{i} \overline{x_{l} u_{j}}\right) d x=\left(\lambda_{i}-\lambda_{j}\right) a_{i j} .
$$

Hence (5.5) becomes

$$
2 \operatorname{Im} \int\left(D_{l}-A_{l}\right) u_{i} \overline{\varphi_{i}} d x=1+\sum_{j=1}^{k}\left(\lambda_{i}-\lambda_{j}\right)\left|a_{i j}\right|^{2} .
$$

Now we estimate the numerator in (5.4). Note that by (5.1)

$$
\operatorname{Im} \int\left(D_{l}-A_{l}\right) u_{i} \overline{\varphi_{i}} d x=\operatorname{Im} \int\left(\left(D_{l}-A_{l}\right) u_{i}+\mathrm{i} \sum_{j=1}^{k} b_{i j} u_{j}\right) \overline{\varphi_{i}} d x
$$

and hence

$$
\begin{aligned}
(\operatorname{Im} & \left.\int\left(D_{l}-A_{l}\right) u_{i} \overline{\varphi_{i}} d x\right)^{2} \\
& \leq\left(\int\left|\varphi_{i}\right|^{2} d x\right)\left(\int\left|\left(D_{l}-A_{l}\right) u_{i}+\mathrm{i} \sum_{j=1}^{k} b_{i j} u_{j}\right|^{2} d x\right) \\
& =\left(\int\left|\varphi_{i}\right|^{2} d x\right)\left(\int\left|\left(D_{l}-A_{l}\right) u_{i}\right|^{2} d x-\sum_{j=1}^{k}\left|b_{i j}\right|^{2}\right) \\
& =\left(\int\left|\varphi_{i}\right|^{2} d x\right)\left(\int\left|\left(D_{l}-A_{l}\right) u_{i}\right|^{2} d x-\frac{1}{4} \sum_{j=1}^{k}\left(\lambda_{i}-\lambda_{j}\right)^{2}\left|a_{i j}\right|^{2}\right) .
\end{aligned}
$$


Combining this inequality with (5.4) and (5.6) we arrive at

$$
\lambda_{k+1}-\lambda_{i} \leq \frac{4 \int\left|\left(D_{l}-A_{l}\right) u_{i}\right|^{2} d x-\sum_{j=1}^{k}\left(\lambda_{i}-\lambda_{j}\right)^{2}\left|a_{i j}\right|^{2}}{1+\sum_{j=1}^{k}\left(\lambda_{i}-\lambda_{j}\right)\left|a_{i j}\right|^{2}} .
$$

Equivalently,

$$
\left(\lambda_{k+1}-\lambda_{i}\right)+\sum_{j=1}^{k}\left(\lambda_{k+1}-\lambda_{j}\right)\left(\lambda_{i}-\lambda_{j}\right)\left|a_{i j}\right|^{2} \leq 4 \int\left|\left(D_{l}-A_{l}\right) u_{i}\right|^{2} d x .
$$

Now we write $a_{i j l}$ instead of $a_{i j}$ to emphasize the dependence on $l$ and define

$$
A_{i j}:=\sum_{l=1}^{d}\left|a_{i j l}\right|^{2} \text {. }
$$

Summing (5.7) over $l$ and using the fact that $V \geq 0$, we obtain

$$
d\left(\lambda_{k+1}-\lambda_{i}\right)+\sum_{j=1}^{k}\left(\lambda_{k+1}-\lambda_{j}\right)\left(\lambda_{i}-\lambda_{j}\right) A_{i j} \leq 4 \lambda_{i} .
$$

Note that $A_{i j}=A_{j i}$. In order to (anti-)symmetrize, we multiply by $\left(\lambda_{k+1}-\lambda_{i}\right)$ and sum over $i$. The resulting inequality is

$$
d \sum_{i=1}^{k}\left(\lambda_{k+1}-\lambda_{i}\right)^{2} \leq 4 \sum_{i=1}^{k} \lambda_{i}\left(\lambda_{k+1}-\lambda_{i}\right) .
$$

This completes the proof of Proposition 2.3 .

\section{ACKNOWLEDGMENTS}

The authors would like to thank Mark Ashbaugh for several helpful remarks. Partial financial support through the SPECT ESF programme for the first and second authors and the Gustafsson Foundation for the third author is gratefully acknowledged. The first two authors appreciate the hospitality of the Isaac Newton Institute Cambridge, where this work was completed.

\section{REFERENCES}

[A] M. S. Ashbaugh, The universal eigenvalue bounds of Payne-Pólya-Weinberger, HileProtter and H. C. Yang. Proc. Indian Acad. Sci. Math. Sci. 112 (2002), 3-30. MR1894540 (2004c:35302)

[AB1] M. S. Ashbaugh, R. D. Benguria, Best constant for the ratio of the first two eigenvalues of one-dimensional Schrödinger operators with positive potentials. Proc. Amer. Math. Soc. 99 (1987), no. 3, 598-599. MR875408 (88e:34039)

[AB2] M. S. Ashbaugh, R. D. Benguria, Optimal bounds for ratios of eigenvalues of onedimensional Schrödinger operators with Dirichlet boundary conditions and positive potentials. Comm. Math. Phys. 124 (1989), no. 3, 403-415. MR1012632 (91c:34114)

[AB3] M. S. Ashbaugh, R. D. Benguria, A sharp bound for the ratio of the first two eigenvalues of Dirichlet Laplacians and extensions. Ann. Math. (2) 135 (1992), 601-628. MR1166646 (93d:35105)

[B] F. A. Berezin, Covariant and contravariant symbols of operators [Russian]. Math. USSR Izv. 6 (1972), 1117-1151 (1973). MR0350504 (50:2996)

[CY] Q.-M. Cheng, H. Yang, Bounds on eigenvalues of Dirichlet Laplacian. Math. Ann. 337 (2007), 159-175. MR2262780 (2007k:35064)

[C] G. Chiti, An isoperimetric inequality for the eigenfunctions of linear second order elliptic operators. Boll. Un. Mat. Ital. (6) 1-A (1982), 145-151. MR652376 (83i:35140) 
[D] E. B. Davies, Heat kernels and spectral theory. Cambridge Tracts in Mathematics 92, Cambridge University Press, Cambridge, 1989. MR990239 (90e:35123)

[HS] E. M. Harrell, J. Stubbe, On trace identities and universal eigenvalue estimates for some partial differential operators. Trans. Amer. Math. Soc. 349 (1997), no. 5, 1797-1809. MR:1401772 (97i:35129)

[H] L. Hermi, Two new Weyl-type bounds for the Dirichlet Laplacian. Trans. Amer. Math. Soc. 360 (2008), 1539-1558. MR2357704

[L] A. Laptev, Dirichlet and Neumann eigenvalue problems on domains in Euclidean spaces. J. Funct. Anal. 151 (1997), 531-545. MR.1491551 (99a:35027)

[LW] A. Laptev, T. Weidl, Recent results on Lieb-Thirring inequalities. Journées "Équations aux Dérivées Partielles" (La Chapelle sur Erdre, 2000), Exp. No. XX, Univ. Nantes, Nantes, 2000. MR1775696 (2001j:81064)

[LP] M. Levitin, L. Parnovski, Commutators, spectral trace identities, and universal estimates for eigenvalues. J. Funct. Anal. 192 (2002), no. 2, 425-445. MR1923409 (2003g:47040)

[LY] P. Li, S.-T. Yau, On the Schrödinger equation and the eigenvalue problem. Comm. Math. Phys. 88 (1983), 309-318. MR701919 (84k:58225)

[LL] E. H. Lieb, M. Loss, Analysis. Second edition. Graduate Studies in Mathematics 14, American Mathematical Society, Providence, RI, 2001. MR1817225 (2001i:00001)

[RS] M. Reed, B. Simon, Methods of modern mathematical physics. IV. Analysis of operators. Academic Press, New York-London, 1978. MR0493421 (58:12429c)

[S] Yu. Safarov, Lower bounds for the generalized counting function. In: The Maz'ya anniversary collection, vol. 2 (Rostock, 1998), Oper. Theory Adv. Appl. 110, Birkhäuser, Basel, 1999, 275-293. MR1747899 (2001d:47036)

[S1] B. Simon, Kato's inequality and the comparison of semi-groups. J. Funct. Anal. 32 (1979), 97-101. MR533221 (80e:47036)

[S2] B. Simon, Maximal and minimal Schrödinger forms. J. Operator Theory 1 (1979), no. 1, 37-47. MR.526289 (81m:35104)

[Y] H. Yang, Estimates of the difference between consecutive eigenvalues, preprint, 1995 (revision of International Centre for Theoretical Physics preprint IC/91/60, Trieste, Italy, April 1991).

Department of Mathematics, Royal Institute of Technology, 10044 Stockholm, SWEDEN

Current address: Department of Mathematics, Princeton University, Fine Hall, Princeton, New Jersey 08544

E-mail address: rlfrank@math.princeton.edu

Department of Mathematics, Imperial College London, London SW7 2AZ, United Kingdom - And - Department of Mathematics, Royal Institute of Technology, 10044 STOCKHOLM, SWEDEN

E-mail address: a.laptev@imperial.ac.uk

E-mail address: laptev@math.kth.se

Department of Mathematics, University of North Carolina, Charlotte, North CaroLINA 28223-0001

E-mail address: smolchan@uncc.edu 